\title{
Anti-PRAME Immunotherapeutic GSK2302032A
}

National Cancer Institute

\section{Source}

National Cancer Institute. Anti-PRAME Immunotherapeutic GSK2302032A. NCI

Thesaurus. Code C116848.

An immunotherapeutic agent targeting the tumor-associated antigen (TAA),

preferentially expressed antigen of melanoma (PRAME), with potential antineoplastic activity. 